9

\title{
Active Catalog: A Knowledge-Rich Design Library Facilitating Information Consumption
}

\author{
Ping Luo and Peter Will \\ University of Southern California /Information Sciences Institute \\ 4676 Admiralty Way, Marina del Rey, CA 90292 \\ E-mail: \{ping,will\}@isi.edu
}

\begin{abstract}
A catalog is a basic resource to a number of people and disciplines ranging from shoppers to companies wanting to sell products. They have traditionally been in paper form and are due for rapid revolution in content and presentation because of the existence of the Internet and the World Wide Web. This paper deals with one component in such a revolution; a component aimed, initially, at aiding the design engineer and later other users.
\end{abstract}

Today's engineering design methodology, in the search for minimum time-to-market, emphasizes reusing existing reliable components wherever possible. This, in turn, requires components to be described in forms useful to the engineer and in forms that help in finding the information about the component in the first place. It also raises new challenges in support for not only efficiently retrieval of information but also for powerful mechanisms for information evaluation and consumption. Although rich in content, today's catalogs and libraries of catalogs and other information are poor in using domain knowledge to help designers to access information in catalogs and libraries during the course of a design. Consumption (in the engineering case) requires the descriptive information to be incorporated not only in documents and drawings but also in simulations of as many modalities as are deemed necessary for performing the engineering task.

In this paper, we describe a prototype system, ActrVe CATALOG, that utilizes a rich body of domain knowledge to facilitate the access and consumption of library contents. The domain knowledge-base system provides a vocabulary that is at a much higher level of conceptualization than that used by traditional database query or the key-word match technique widely employed by Web applications. Based on the knowledge of the domain and the meaning of the queries specified in this vocabulary, a search engine accesses 
library components that satisfy the "semantics" defined by the queries. In addition, ACtive CATALOG, suggested by its name, adds to traditional library contents such as textual and image information, a rich set of models of different modalities. The system, in turn, allows a designer to down-load models, viewers, running code or simulation code to evaluate dynamic and behavioral aspects of a component in a systems environment via interactive simulation before committing the component for prototype or production use.

Active Catalog methodology of use supports a new "Try-Before-You-Buy" methodology.

\section{Keywords}

Knowledge-based Information Retrieval, Semantic Network, Database-Knowledgebase Integration, Design Library, Simulation-based Design.

\section{INTRODUCTION}

Today's design environments must provide the designer with, in addition to traditional tool, libraries and catalogs of reusable components in relevant design domains (Will 1991). AUTODESK's Parts Library (PartSpec) for mechanical design and SmartModel Library (PartSpec) for circuit design are examples. The major benefits of using libraries and catalogs are well-known: the products, designed with reusable components, are cheaper, better and faster-to-market. With the ability to save partial designs, designers could cache information for future applications and thus, bootstrap their effort.

Most of the benefits of catalogs and libraries are based on a major prerequisite: that the information is easily and conveniently accessible to the designer (i.e., it is reachable and usable). This assumption does not naturally hold in general. Although rich in content, today's libraries are poor in access and their contents are designed to be consumable by a human reading a document or looking at an image. The design domain is one where the information is to be automatically consumed by a computer and is harder. This poor situation would be improved a) by using domain knowledge to give designers easier access to library contents during the course of design and b) by improving the means of consumption of the information by computers after it is retrieved.

There exists a gap between the high-level, conceptual mental model of a part needed by a designer and the low-level, physical query that retrieves the needed information from a library. Too often, the designer is required mentally to map the description of a desirable component for solving a particular problem onto a set of searchable attributes recognizable by the library/catalog. That is, the user needs essentially to understand the "schema" of the libraries and the searchable technical attributes of the library contents before mapping the need into a set of queries. This mapping is not trivial and imposes a major mental burden on designers, deflecting them from focusing directly on solving their design problem. Using the information from the library/catalog is equally onerous and often involves manually transcribing the information into the local design tool environment. This is especially true when the data must be entered into CAD or be prepared for consumption by distributed simulation environments. 
In this paper, we describe a prototype system, ACTIVE CATALOG, that addresses the above problems by using a rich body of domain knowledge to describe the library components, adding to and annotating traditional library information and aiding the use of the retrieved information in CAD applications. Designers, using the system, can search library contents using the familiar terminology of the design domain. A search engine, based on the knowledge of the domain and the meaning of the queries specified in the domain vocabulary, accesses library components that satisfy the functionality meant by the queries, rather than by matching the keywords or synonyms in the queries. ACTIVE CATALOG, suggested by its name, adds to traditional library contents (such as textual and image information), a rich set of models of different types that, in turn, allow a designer to evaluate dynamic and behavioral aspects of a component in a system via interactive simulation built from or using down-loaded models, viewers or even applets of simulation code.

The goal of this research is to improve both design environments and the general consumption of information found in catalogs and libraries. We approach this goal, done here in the context of electro-mechanical engineering design, by addressing several fundamental HCI, cognitive issues. As noted, there can be a gap between the user's mental model of a part and the set of queries needed to access it. We humans cannot perform high quality reasoning and provide correct judgement if there exists no direct mapping between a real world object and our mental model of that object; and we are much less productive when we are diverted by too many distracting tasks; we cannot keep track of many threads or recall all what we know. Last, but not least, we cannot access material if we are not aware of its existence. We aim at providing technologies that enable designers to dedicate their mental resources to the design problems at hand and are using a knowledge-based approach to do it.

The rest of the paper is organized as follows. We first give an overview of the system architecture by illustrating system substrates. We then describe in detail the key relevant system components. These components include a semantic network of the domain that enables a high-level, flexible, and user-centered approach to information query; a knowledge-based search mediator that hides distributed and heterogenous information sources from a user; and a set of middle-ware that enables a distributed simulation environment to assist in evaluating the dynamic and behavioral aspects of a piece of information retrieved. One section will be dedicated to each of these components, followed by reviewing of related work and our future directions.

\section{OVERVIEW OF THE SYSTEM STRUCTURE}

An ACtive CATALOG, as the name suggests, is a normal catalog enhanced by information that is dynamic in one or several dimensions. The word active is used to connote the idea that the information is not static, pre-canned sequences of descriptions of the data in the catalog, (nor merely regular multimedia: whether sound, image or movie) but may itself contain information that can, in turn, compute the required answer or even be used to compute the answer. The computation can be done either: 
- in the output stage of the search and thus be dynamically tuned and delivered, or,

- in the recipient's local or distributed computing environment by delivering a program (perhaps a JAVA applet) for local use, or,

- used to configure the users environment.

In a design environment this takes the form, in the first implementation, of using components and parts described by a set of models of different modalities to make system simulation models and thus help the engineer produce better products. The types of models include, for example, a mathematical model, various kinds of simulation models, an engineering drawing, a 3D geometrical model, a transfer function model, kinematic and dynamic models, textual and semantic descriptions, a set of information viewers and even simulation code.

The major components of an Active Catalog (FIGURE 1) are:

- a semantic model of a domain,

- a set of parts in the domain described as catalog entries, for each part a set of appropriate models in the modalities mentioned,

- a set of databases that stores the models,

- a search engine that recognizes users' queries specified in a vocabulary given by the semantic model of the domain, and

- a set of helper applications for viewing the information retrieved.

Also provided is a set of application programming interfaces (API) to facilitate the integration of a parts from a catalog, or even the catalog itself, into engineering and electronic commerce environments.

A user is anticipated to be an engineer doing a new design, a repair/maintenance person maintaining a product or a potential buyer wishing to test a component before buying it. This "Try-Before-You-Buy" paradigm may well be the most important outcome of the work described here and has wide applicability. The user, as shown in FIGURE 1, can either browse catalog contents or issue high-level conceptual queries for the desirable information. A knowledge-based search engine, taking as input the user's high-level query and the domain knowledgebase, matches catalog contents with the semantics of the query. When a piece of information is returned by the search engine, the system extracts retrieved information of different types and dispatches it to the corresponding software in the users's environment such as simulators, spread-sheets, helper applications and viewers that handle the appropriate modality. When necessary, a distributed simulation environment can be automatically configured and executed.

We will focus in the following sections on the capability of an Active CATALOG in providing knowledge-based catalog and library support to the engineer.

\section{THE DOMAIN}

The domain of pumps will be used as an example through out the remainder of this paper. 


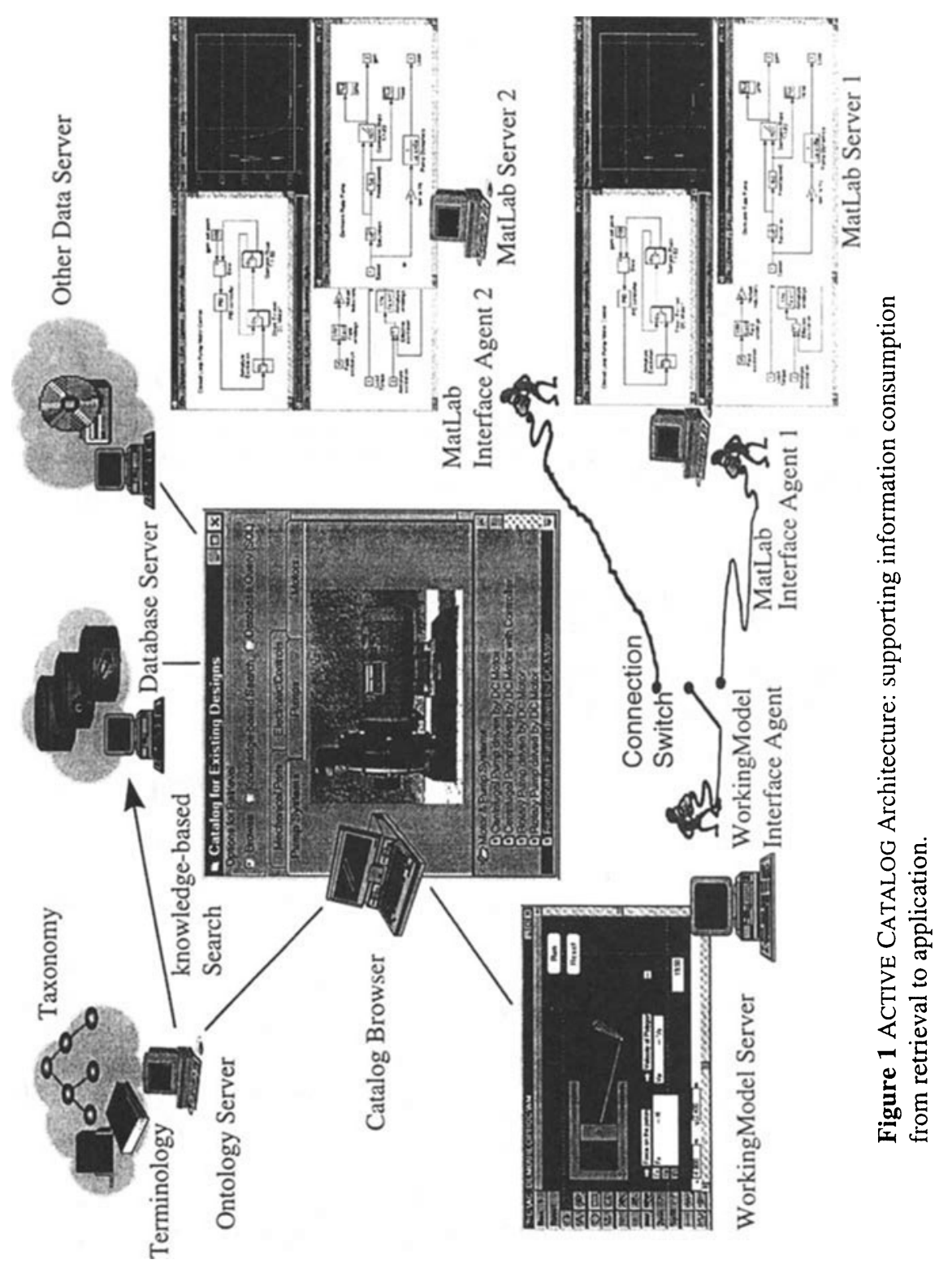


This domain was chosen for study in the context of simulation based design of electromechanical systems found in ship design and maintenance. Although the description of the work that follows is specific to pump systems, the technologies and mechanisms developed are domain independent and have wider applicability. The use of the system may be illustrated with reference to the example shown in Figure 1. There the user is faced with a problem he or she needs a pump to solve an engineering problem. The user enters the catalog module and describes the query in any one of the several methods (described later). The system conducts a knowledge based search of a variety of catalogs available locally (or on the WWW soon) and then returns components matching the description including the description of the pump drive requirements, the geometric size, weight, connector needs etc. The user then does a Try-Before-You-Buy action by choosing to test the objects (the pump and a similarly chosen motor for example) returned in response to the query in a chosen simulation environment. The object describing the returned part is then examined and the various parts of it sent to the appropriate simulator. In the pump/motor example used here, mathematical and transfer function simulation models of both pump and motor are sent to MatLab/SimuLink (MatLab) for dynamics analysis while kinematic models for simulation of the pump pressure and stroke interactions are sent to WorkingModel (WorkingModel).

\section{SEMANTIC NETWORK DESCRIBING THE DOMAIN}

The key to knowledge-facilitated search for information is the existence of a semantic network (Brachman 1991) for the domain. It needs to describe:

- taxonomies of the domain objects (parts and components in the catalog or library);

- their attributes, values and value constraints;

- taxonomies of the attributes, values and value constraints; and

- relationships among these objects, attributes, and attribute values and value constraints. There are few semantic models of any domain in existence at present. The Ontology project at Stanford (Farquhar et al. 1995) provides a framework for unifying and disseminating models, but until a corpus of semantic models exist each new domain will have to be constructed as needed. Tools for the construction of semantic models are needed.

In the case here, the semantic network is modeled in Loom (MacGregor 1990), a knowledge representation system language and environment developed at ISI for constructing intelligent applications. The Loom system provides deductive support for the declarative portion of the Loom language. Declarative knowledge in Loom consists of definitions, rules, facts, and default rules. The semantic model of pumps consists mainly of definitions and matches Loom's power.

\subsection{Main Taxonomy for Pumps}

The main taxonomy of the semantic network describes pump types. It is similar to a typical product classification found in a traditional catalog, except that the classifications 


$\begin{array}{lll}\text { Type Hierarchy } & \text { Value Constraint } & \text { Attribute Hierarchy } \\ & \text { Hierarchy } & \\ \text { Centrifugal Pump } & \text { Construction } & \text { Impeller Structure } \\ \text { Axial Flow Centrifugal Pump } & \text { Casing Construction } & \text { Impeller Structure By Flow Direction } \\ \text { Mixed Flow Centrifugal Pump } & \text { Coating And Lining Construction } & \text { Impeller Structure By Openness } \\ \text { Radical Flow Centrifugal Pump } & \text { Impeller Construction } & \text { Rotor Structure } \\ \text { Screw Centrifugal Pump } & \text { Wearing Ring Construction. } & \text { Bearing Structure } \\ \text { Special Pump } & \text { Driver } & \text { Circumferential Piston Structure } \\ \text { Electromagnetic Pump } & \text { Brake Horsepower } & \text { Diaphragm Structure } \\ \text { Gas Lift Pump } & \text { Driver Position } & \text { Flexible Member Structure } \\ \text { Hydraulic Ram Pump } & \text { Driver Type } & \text { Gear Structure } \\ \text { Jet Pump } & \text { Pitch } & \text { Lobe Structure } \\ \text { Pitot Pump } & \text { Installation } & \text { Piston Structure } \\ \text { Screw Centrifugal Pump } & \text { Geometry } & \text { Screw Structure } \\ \text { Viscous Drag Pump } & \text { Mounting } & \text { Vane Structure } \\ & \text { Weight } & \end{array}$

Figure 2 Sample taxonomies of pump types, attributes and value restrictions from ACTIVE CATALOG's semantic network of the domain.

are much more extensive.

The pump taxonomy is organized as a graph rather than a tree structure. This provides multiple paths to a specific pump type and is implemented using LOOM's multiple inheritance features. At the top levels of the taxonomy are a few abstract type-definitions for pumps, such as Kinetic Pumps and Positive Displacement Pumps, with Special Pumps, Peripheral Pumps and Centrifugal Pumps being subclasses of Kinetic Pumps, and Rotary Pumps, Blow Case Pumps, and Reciprocating Pumps being subclasses of Positive Displacement Pumps. In the middle levels are a rich set of abstract classes defining varieties of pump classes. At the bottom levels, a large number of pump classes are defined by combining types from top and middle levels. For example, Reciprocating Single Acting Power Multiplex Pumps are defined as sub-class of Reciprocating Pumps, Single Acting Power Pumps, and Multiplex Pumps. Figure is a small sample of the present pump taxonomy, which consists of more than one hundred pump types, in our semantic net, which has over a thousand entities.

There are several benefits to making a taxonomy with a graphical structure. Since most bottom-level, specific pump types have multiple parents, the users of the taxonomy can reach the same target types via different paths. This enables the user to issue partially specified queries in terms of known pump types/features without sacrificing the capability of reaching the target. The taxonomy used in Active CATALOG does not require its user to know and remember the exact types of the pumps needed. For example, a Reciprocating, Single Acting, Power Multiplex Pump is reachable via any of (or combination of) the following pump types: Reciprocating Pump, Single Acting Power Pump, Multiplex Pump, and all the pump types that are the parents (in the type hierarchy) of these three types. 


\subsection{Main Taxonomy for Attributes and Value Constraints}

Attributes describe properties of the objects in the semantic network. A unique feature of attributes is that, like pump types, they are organized hierarchically in the semantic network in order to facilitate query specification and reasoning. For example, the attribute, Pump Structure, is a high-level abstract attribute, with several sub-attributes: Propeller Structure, Prime Mode, Internal Structure, and so on. An attribute taxonomy allows queries to ask loosely for the Structure of a pump being of type Self-Priming or Peripheral rather than for the query to specifically ask for Priming Structure to be SelfPriming and Internal Structure to be Peripheral respectively.

A value constraint specifies the range of all possible values for an attribute either in the context of a pump type or in a context-free form. All value constraints constitute the space of attribute values. As in types, the value constraints are organized hierarchically into a taxonomy. For example, the value constraint for the attribute, Casting Material of Pumps, is Solid Material, which could be Cast Iron, High Silicon Iron, or Naval Bronze.

By combining the above taxonomies, the user can construct a specific query such as, find Pumps whose Casting Material is Bronze, or a more conceptual query, Pumps whose Structure is Corrosive-Resistant Material. (Bronze is also one of its sub-classes). Of course the first query is more specific and precise, however, it requires more knowledge about pumps and more skill to specify; whereas the second is easier to construct but will more likely return a larger set in the result,. Although both forms are needed, the second one is preferred in many situations since it requires less initial specificity. In general, it is much harder to generate a correct set of results by one precise query than to achieve the same set by filtering from a larger set of candidates (if the size of the set of candidate is manageable).

Although seemingly more flexible and more user-friendly than traditional information retrieval, the queries so far are still described in terms of technical attributes. That is, the user is required to transform domain problem into technical attributes and terminology and to use technical-oriented vocabulary to construct queries. Users need to be able to state a query in the vocabulary of the applications domain.

\subsection{Semantic Network}

A semantic network, woven with the above (types, attribute, and value constraint) taxonomies, declaratively describes knowledge traditionally used by experienced engineers working in the domain. A declarative representation of such knowledge is easily sharable, transferrable, and maintainable; it can be used by computer programs to automate sophisticated, routine tasks that otherwise would have to be performed by the user. One such task is the mapping of a description of an engineering part or component that satisfies a domain need into a set of representations and queries that can be recognized and acted upon by the information sources storing the corresponding parts and components.

Our current semantic network in Active Catalog captures a sub-set of the total knowledge of pump systems, comprising descriptions of pumps, motors used for driving 


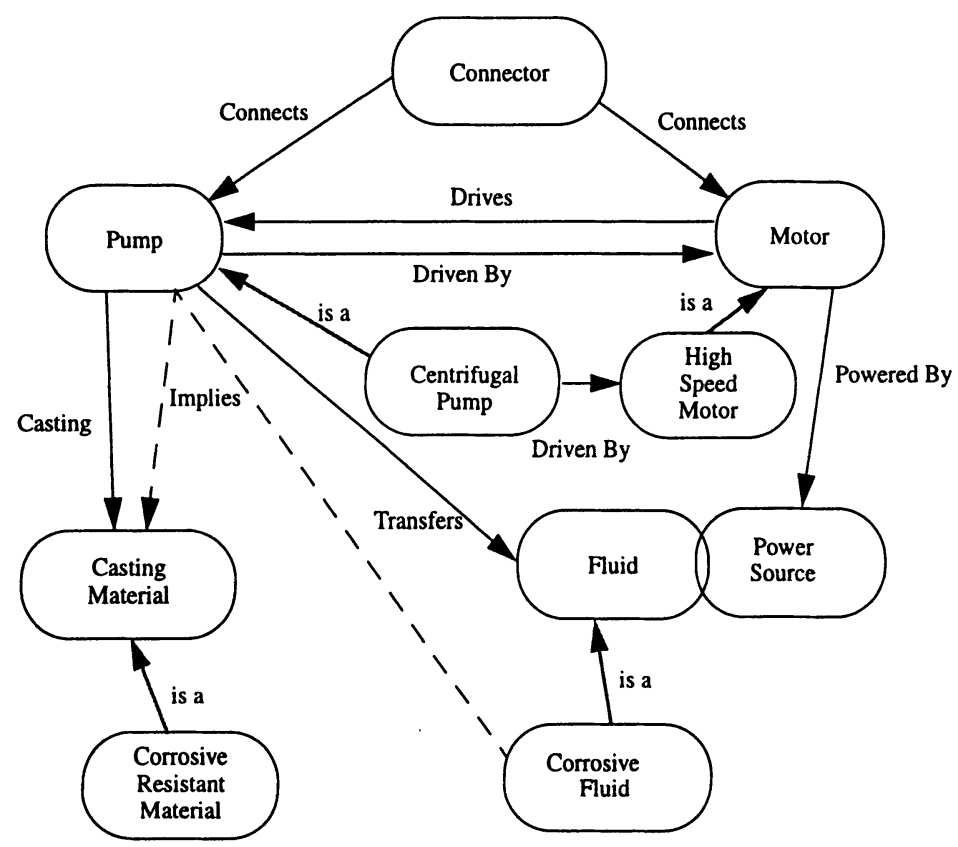

Figure $3 \mathrm{~A}$ fraction of pump knowledge from the semantic network in ACTIVE Catalog.

them, their connections, and their applications.

Figure graphically depicts a piece of the semantic network describing the relationships between pumps and motors and the relationships between the type of fluid to be transferred and the casting materials for pump.

To avoid a lengthy discussion, we will not describe here every detail about the model but will focus only a few interesting points. First, the model has an open structure. It provides a template for expanding into a much richer and detailed network depending on the need of the application. For example, a model of fluid type, (corrosive, water-based, oil-based, rheological coefficient etc.), power source, casting material, corrosive resistant casting material, or connector can be added to enrich the depicted model.

Second, the model captures knowledge about applications. For example, the network contains a piece of deductive information that if a pump is used to transfer corrosive fluid, then the pump's material should be corrosive-resistant. In fact, our network provide a set of different types of corrosive fluid, corrosive resistant materials, and a set of correspond implication rules. Users can then directly describe the use of the pump in application 
terms, such as find a high capacity pump for transferring sea water or find a motor that drives a high-capacity centrifugal pump, without having to know the exact technical terms for describing pump parameters.

Third, the network facilitates information access even if the existence of the information is unknown to the user. When a new type of material becomes available we make the new material retrievable by simply adding the model and implication rules for the new material without touching other parts of the network. All the changes are then transparent to the end user. That is, even in the case of repeating an old query, the system will automatically reply with the new material even though the user may not be aware of its existence and its availability.

Fourth, the semantic network captures the underlying semantics of the domain and is sharable across domains.

\section{USER-FOCUSED QUERY}

The semantic model provides a rich vocabulary for use in constructing queries. Therefore, users are able to retrieve the same set of information via many channels:

- by describing attributes and values in technical terms (the traditional way of searching for information),

- by describing the applications at hand in user domain terminology, or

- by, via indirection, by describing the problem to be solved.

For example, the following queries could be semantically equivalent in a given data source of some domain:

- a centrifugal pump with bronze casting and propeller,

- a pump that pumps seawater, or even

- "we need to pump out seawater" (taking into account the context is in ship design).

Similarly, users can ask for motors by giving pumps as constraints.

User-focused query is a key concept in accessing today's ever-evolving information sources. The amount of information and its availability via networks offers great potential to leverage the work of others. However, in today's information systems too many things (such as the physical locations of information sources, contents of the sources, information formats from the sources and structures of the sources) are changing too often and the user is unnecessarily and undesirably exposed to these changes. Userfocused query provides a solution to overcome this problem. In the Active Catalog environment, the user is insulated from unnecessary change by a body of stable domain knowledge. A user's query will have a longer period of persistence or validity in spite of changes in the content or even the physical location of the data.

\section{SEARCH ENGINE}

The semantic network is declarative as mentioned previously. The declarative nature is 
necessary for being able to share the domain knowledge between human users and the computer. The semantic model provides a vocabulary that is close to (if not the same as) the one employed by the user in daily work and a structure that captures the nature of the expert knowledge about the domain. The model explicitly describes attributes, their values and their value constraints, as well as the relationships between the objects modeled are explicitly. This allows the model be used by a system of reasoning modules such as one that automates the translation of a semantic query into corresponding queries of physical data repositories.

Active Catalog uses SIMS (Arens et al. 1993), (being developed at ISI) as its knowledge-based search engine. SIMS is an intermediate layer - a mediator - between information sources and humans users or applications programs. Queries to SIMS are in a uniform language, independent of the distribution of information over sources, of the various query languages, the location of sources, etc. SIMS determines which data sources to use, how to obtain the desired information, how and where to temporarily store and manipulate it, and how to maintain an acceptable level of efficiency in performing its task.

At the top level a query in Active CATALOG is translated into a SIMS query using the terminology of the semantic network. SIMS converts the initial query into a set of semantically-equivalent queries to the physical information sources. SIMS to do this needs to know the structure of the information sources and the mapping from semantic terminology into physical data sources. The structural information of the data sources can be modeled easily (in fact, the major part of the model can be generated automatically by querying the schema information of the data sources); the KB-DB mapping at present needs to be hand crafted,; better tools are needed here.

With the three pieces of model, the domain model, the information source schema model, and the KB-DB mapping model, SIMS performs reasoning on a semantic query (such as changing "seawater pump" into "bronze casting material"), partitions a potentially complex semantic query into many small pieces and transforms the small pieces into actual queries to the physical information sources, taking into account the heterogenous and distributed nature of the sources, the efficiency of database joining, parallel accessing information sources, and redundant information storage such as mirroring and partial overlapping.

\section{INFORMATION CONSUMPTION}

Major effort in ACTIVE CATALOG has been focused on aiding its users (in the example, engineering designers) in evaluating the information returned in response to their semantic queries. When a piece of information is retrieved, based on its type, the system dispatches the information to an appropriate viewer or consumer. For example, if the information is a engineering drawing in AutoCAD (AutoCAD), the system invokes the AutoCAD environment and loads the drawing; if the information is a differential equation or transfer function described by a MatLab model, MatLab/ SimuLink is brought up with the model inserted; if a brochure, the presentation environment is run. 
In a more sophisticated simulation system, for example, when a piece of information is multi-modal, the system separates the total retrieved model object into its constituent modalities and then dispatches each piece of the model to its corresponding tool whether in a local or distributed environment.

The use of the system may be illustrated with reference to the example shown in Figure 1. As described previously the user queries the system and an appropriate part is returned. The object describing the returned part is then examined and the various parts of it sent to the appropriate simulator. In the pump/motor example used here, mathematical and transfer function simulation models of both pump and motor are sent to MatLab/ SimuLink for dynamics analysis while kinematic models for simulation of the pump pressure and stroke interactions are sent to WorkingModel. Each system has its own display and animation features.

The working system was implemented on a set of distributed machines using software agents running on top of DDE and TCP, and will only be described briefly here. Executing the mathematical model in its corresponding environment requires the system to automate a sequence of tasks intelligently. The system first sends out a message to a Broker (actually running on a different machine to emphasize the distributed nature of the work) requesting for MatLab service. The Broker searches its database for pre-registered hosts capable of providing the service. The search result, a host candidate meeting the specification, is returned to the requesting module that, in turn, contacts the named host for service. Upon establishing a connection to the host, Active CATALOG sends the mathematical model to the server, sets up proper communication channel for data exchange between MatLab and WorkingModel and executes the simulation. Thus, the models are executed in a distributed environment configured at runtime and hidden from the user.

The user then examines the results, selects other models, other pumps, or motors, or combinations of both, until an acceptable design solution is reached. The satisfactory parts are then "bought". Currently we plan to integrate with FAST (FAST), an electronic commerce system operating at ISI to implement real purchases.

The Active Catalog system implements "Try Before You Buy", a powerful, new paradigm of information access. This paradigm, although illustrated here in the domain of pump/motor systems, is not limited to this particular domain or solely to engineering design. Instead it is applicable to a wide range of applications as diverse as education and electronic commerce.

\section{RELATED WORK}

Engineers usually work with catalogs, acquired over the years, that contain descriptions of parts and sub-systems. The catalogs form not only the repository of information describing the particular part but also form pointers to sources of the part. (An obsolete catalog may still be useful in that it points to a source, engineers keep them for this reason). Current, up-to-date, catalogs contain descriptions of parts at a detail sufficient not only for purchase but also for simulation use. The catalog often contains a picture of 
the part, a set of descriptors (size, weight, speed and other appropriate specifications, drawings etc.). Often included in the catalog is context information... "if you use this part you need to buy this widget to make it work". Sometimes the catalog contains a short technical paper or applications note that tells how the part has been used in a conventional or sometimes unconventional manner. Searching for a part is usually a manual operation first to find the catalog and a manual operation via the catalog index to then find the part. The process could be improved by access to a wider field of information such as that provided by the WWW. In addition AcTIVE CATALOG provides improved tools for knowledge based search and dynamically configured simulation both of which add useful capability.

Catalogs are becoming on-line. For example, see the work of and product provided by IndustryNet (IndustryNet), PARTNET (PARTNET) et al. Search of these Net-based catalogs is mostly by keyword match, with searchable attributes and their values in technical terms. Their data can be centralized or federated databases depending on the provider.

AUOTDESK has produced a CD-ROM of drawings of mostly single parts designed in their AUTOCAD product that can be down- loaded into a users system for reuse in the drawing being produced.

Logic Modeling Group (LMG), a part of Synopsys, Inc. provides libraries of simulation models for VLSI parts. These models work with the major VLSI CAD systems. The provision and use of models that can be inserted into CAD systems powerfully improves the user's ability to produce good designs at a faster rate than normal. The design can be proven before any component is purchased and often eliminates the need for building several sequential physical models.

Model-based design in the mechanical design domain is rare. Most mechanical "design" systems are actually drawing systems. The Active CATALOg concept was invented to rectify this omission.

\section{FUTURE WORK}

Developing a semantic network that captures the true nature of all objects in the universe of engineering design is impossible. It is possible and very desirable to build semantic networks for focused domains. This will significantly leverage the ability of designers in accessing information. The construction of a close-to-complete semantic model of a small domain requires a great amount of effort in knowledge acquisition and engineering. The pump network had 300 classes of pumps, 3000 attributes and took three months to build. The tools for constructing such networks need improvement but once done, the knowledge is expected to last a long time, Cooperative efforts with other groups to build and share semantic networks are important. Since Active Catalog has already demonstrated the need of such semantic models, we will next test the extensibility of our framework by utilizing existing domain knowledge-bases and ontology work from other institutes and industry and will focus on middle-ware technology to coordinate and reconcile knowledgebases. 
Our current implementation of the facilitator for simulation-based evaluation relies on a body of knowledge about simulation, models, tools, interfaces and data exchange protocols. However, the knowledge is implicitly represented by fragments of programming code. This will be converted to explicit representations. The need for explicitness about servers and their capabilities has already surfaced during server selection and in the negotiation and contracting for the WorkingModel and MatLab services.

\section{CONCLUSION}

Active Catalog, brings a conceptually new idea to the design domain in the specific and to electronic commerce in general. It gives an electronic version of "Try Before You Buy" where you "try" in whatever modality is correct for "your" domain. It also supports a wide variety of search mechanisms including functional search and supports the use of a wide variety of models and modelling systems. The consumption of catalog items in a design or modelling environment is predicated on an adequate set of information interchange mechanisms. These are provided by agent based communicators.

Active Catalog facilitates the work of an engineer or other user by using intensive knowledge of the domain to enhance the retrieval of useful information more easily. and for sifting through the information by using tools, including some for the configuration of simulation environments. It contributes to knowledge intensive $\mathrm{CAD}$ and benefits general information accessing (from retrieval to consumption) in a variety of dimensions:

- Open-ended and modularized domain knowledge models for ease in bidirectional knowledge sharing. As discussed previously, the semantic network in ACTIVE CATALOG gives expanded coverage and enriched detail our current knowledgebase by being able to use external plug-in knowledgebases. In turn, its knowledge bases serve as stand-alone knowledge modules that can easily be adapted into other knowledgebases and semantic networks.

- High-level, flexible, conceptual queries provide user-focused information retrieval. Our knowledge-enabled query allows users to specify needs in a domain vocabulary. The query can be partially specified without compromising the capability of retrieving the target information; the query can be specified in some cases by describing the application and the problem without the needing to know the detailed technical terminology. The support of user-focused queries insulates the user from many undesirable details such as storage format changes, specific terminology used by different pieces of information, and issues of mapping between different terminologies and different mental models.

- Highly automated task environment configuration. ACTIVE CATALOG currently implicitly captures a body of knowledge about tools for information viewing and evaluation. This knowledge goes the current capabilities of Web Browsers and uses procedural knowledge to configure the distributed simulation environments needed for information viewing and evaluation as the need arises.

- Integrated retrieval and evaluation environment offering a seamless information 
flow. Active Catalog, using knowledge intensive configuration agents, integrates information retrieval and evaluation (as in the simulations described here) as a seamless whole, off-loading some distracting time-consuming tasks from the user.

We believe that an information-rich environment is a necessity for many users in the current Information Age and especially for engineers who have advanced demands. Information-richness, in itself, may not necessarily, automatically facilitate the work of a user; in fact and ironically, many such systems distract the user. The key effort of ACTIVE CATALOG is to provide a set of enabling technologies that allow the future information user to focus on the problem to be solved, not on the computing details of how to run the models and the environment.

\section{ACKNOWLEDGMENT}

We wish to thank Houchang $\mathrm{Lu}$ and Shivanand Bhajekar for participating in the implementation of the system. This work was supported by DARPA MADE Program under contracts J-FBI-95-159. The contents represent the opinions of the authors, and do not reflect official positions of DARPA or any other government agency.

\section{REFERENCES}

Arens, Y., Chee, C.Y., Hsu, C.N. and Knoblock, C.A. (1993) Retrieving and Integrating Data from Multiple Information Sources. International Journal of Intelligent and Cooperative Information Systems. Vol. 2, No. 2. Pp. 127-158.

AutoCAD. Autodesk, Inc., 111 Mclnnis Parkway, San Rafael, CA 94903, USA.

Farquhar, A., Fikes, R., Pratt, W., and Rice, J. (1995) Collaborative Ontology Construction for Information Integration. Knowledge Systems Laboratory Department of Computer Science, KSL-95-63.

FAST. http://info.broker.isi.edu/fast.

IndustryNet. http://www.industry.net.

LMG. Logic Modeling Group, Synopsys, Inc. 19500 NW Gibbs Drive Beaverton, OR 97006, USA.

MacGregor, R. (1990) The evolving technology of classification-based knowledge representation systems. In Principles of Semantic Networks: Explorations in the Representation of Knowledge (ed. J. Sowa), Morgan Kaufmann.

MatLab. The MathWorks, Inc., 24 Prime Park Way, Natick, MA 01760-1500, USA.

PARTNET. http://www.part.net.

PartSpec and MaterialSpec. Autodesk, Inc. 111 Mclnnis Parkway, San Rafael, CA 94903, USA. 
R.J. Brachman Ed. (1991) Principles of Semantic Networks: Explorations in the Representation of Knowledge. The Morgan Kaufmann Series in Representation and Reasoning.

Will, P.M. (1991) Simulation and Modeling in Early Concept Design: An Industrial Perspective. Research in Engineering Design. V3, N1. Springer International.

WorkingModel. Knowledge Revolution, 66 Bovet Road, Suite 200, San Mateo, CA 94402, USA.

\section{BIOGRAPHY}

Dr. Ping Luo is a research computer scientist at Enterprise Systems Integration Division, USC/ISI. His work fells into a wide spectrum of research areas: Human-Computer Interaction, User-Interface design tools and environments, Software Engineering, Knowledge-based systems for information retrieval, tools and systems integration, and Electronic Commerce.

Dr. Peter Will is Director of the Enterprise Systems Division at USC/ISI, He directs programs on design of high performance processors, low power systems, MicroElectroMechanical Systems, electronic commerce, active catalogs, manufacturing systems and quantum computing. Earlier, he managed ISI's High Performance Computing and Communications Division. Prior to joining ISI, Will was with IBM's Yorktown Research Lab as an robotics and vision researcher and manager, with Schlumberger as a research director, and at HP Labs as the Director of Measurement Systems and Design Systems. Will has over 50 publications and 10 patents, and he has been active on government committees, most notably as chair of three NSF advisory committees, as Chair of the recent National Academy study on Information Technology in Manufacturing, as a member of the Computer Science and Technology Board of the National Research council and as a participant and leader of several DARPA ISAT studies. In 1990 he was awarded the international Engelberger Prize in robotics. 